Pacific Journal of Mathematic 


\title{
LOCALLY QUASI-UNMIXED NOETHERIAN RINGS AND IDEALS OF THE PRINCIPAL CLASS
}

\author{
L. J. RATLiFf, JR.
}

It is proved that a Noetherian ring $R$ is locally quasiunmixed if and only if, for each ideal $B$ of the principal class in $R,\left(B^{i}\right)_{a}$ is height unmixed, for all $i>0$, where $I_{a}$ denotes the integral closure in $R$ of an ideal $I$ in $R$. Three applications are given.

1. Introduction. The main theorem in this paper (mentioned above) gives a characterization of locally quasi-unmixed Noetherian rings. Since such rings occur in many investigations in commutative algebra and algebraic geometry, it is desirable to know as many properties of such rings as possible. The above theorem gives one such property, and that such rings have this property is a new result, and until now was not known to hold even in a regular local ring or a complete local domain. Since the concept of the integral closure of an ideal has proved to beu seful in numerous research papers, for example [1], [2], [5], [6], [14], [15], [16], [17], and [20], the fact that ideals of the principal class in such rings have the property stated in the theorem should be of importance in future investigations. On the other hand, there are many open problems concerning the chain conjectures (see [11, §2] for the relationship of quasi-unmixedness to the chain conditions for prime ideals, and see the introduction of [12] for some of the open problems in this area), so properties of locally quasi-unmixed Noetherian rings which characterize such rings are of interest and importance in solving these problems. Beyond this, the above theorem has some interesting applications, and some of these are given in $\S 3$ of this paper.

A brief description of this paper will now be given. The proof that a locally quasi-unmixed Noetherian ring has the above stated property is given in 2.12, and the material preceding this result consists mostly of definitions and a review of known results concerning the definitions. After proving 2.12, a number of corollaries are given, among which are the following (where $R$ is a locally quasiunmixed Neotherian ring): (a) The integral closures of the powers of certain other ideals in $R$ are also height unmixed 2.14; (b) The same result holds in each ring $S$ such that $R \subseteq S \leqq R^{\prime}$, where $R^{\prime}$ is the integral closure of $R$ in its total quotient ring (that is, if $B$ is an ideal of the principal class in $S$, then $\left(B^{i}\right)_{a}$ is a finite intersection of primary ideals and is height unmixed, for all $i>02.20$ ); and, (c) If $B$ is an ideal of the principal class in $R$ and $I$ is an ideal 
in $R$ such that $I$ is not contained in any minimal prime divisor of $B$, then, for all $i>0$ and $n>0, B^{i}: I^{n} \subseteq\left(B^{i}\right)_{a} 2.19$. After these corollaries, the converse of 2.12 is considered. Its proof is difficult, since it is first necessary to establish two preliminary results (which are of some interest in themselves): ( $\left.a^{\prime}\right)$ a result concerning the prime divisors of an ideal generated by a nonzero-divisor in the integral closure of a Noetherian ring 2.22; and, $\left(b^{\prime}\right)$ a result concerning the prime divisors of $\left(B^{i}\right)_{a}$ where $B$ is an arbitrary ideal in an arbitrary Noetherian ring 2.24 and 2.25. Once these results are known, the proof of the converse of 2.12 is given 2.29. Finally, examples are given to show that: $\left(a^{\prime}\right)$ does not hold in nonintegrally closed Noetherian rings 2.28 (a); and, the two equivalent statements in the theorem are not equivalent (in the local case) to the existence of an ideal $B$ of the principal class in $R$ such that height $B=$ altitude $R-1$ and $\left(B^{i}\right)_{a}$ is height unmixed, for all large $i 2.28(\mathrm{~b})$.

In $\S 3$, three applications of 2.12 are given. The first application shows that a fairly easy proof of a theorem due to E. Böger can be given 3.5 (the theorem being a generalization of a deep result due to D. Rees), and then some extensions of the result are given 3.63.9. The second application has to do with the prime divisors of certain ideals related to an ideal of the principal class in an analytically unramified and locally quasi-unmixed semi-local ring 3.11, and the third application shows that if $R$ is locally quasi-unmixed and $B=\left(b_{1}, \cdots, b_{k}\right) R$ is an ideal of the principal class, then $\left(B^{i}\right)_{a}: b_{j} R=$ $\left(B^{i-1}\right)_{a}$, for all $i>0$ and $j=1, \cdots, k 3.13$.

2. Two characterizations of locally quasi-unmixed Noetherian rings. All rings in this article are assumed to be commutative rings with a unit element, and the terminology is, in general, the same as that in [4]. However, to keep the article reasonably self-contained, a number of definitions are given.

The main purpose of this section is to prove Theorem 2.29 which gives two characterizations of a locally quasi-unmixed Noetherian ring in terms of a property of ideals of the principal class. The proof that this property holds in such rings is given in 2.12 and is quite straightforward using known results, once the definitions (and some auxiliary definitions) have been given. We begin with the following definition.

Definition 2.1. Let $B$ be an ideal in a ring $R$. Then the set $B_{a}$ of elements $x$ in $R$ such that $x$ satisfies an equation of the form $x^{n}+r_{1} x^{n-1}+\cdots+r_{n}=0$, where $r_{i} \in B^{i}$, is the integral closure of $B$ in $R$.

Clearly $B \subseteq B_{a} \subseteq \operatorname{Rad} B$, and it is known $[6, \S 6]$ that $B_{a}$ is an 
ideal in $R$ which is integrally closed in $R$ (that is, $\left(B_{a}\right)_{a}=B_{a}$ ).

To avoid continual repitition, the following notational convention will be used throughout this paper.

Notation 2.2. If $R$ is a ring, then $R^{\prime}$ will denote the integral closure of $R$ in its total quotient ring.

Lemma 2.3. (cf. [8, Lemma 1].) Let $b$ be a nonzero-divisor in a ring $R$. Then $(b R)_{a}=b R^{\prime} \cap R$ and an element $c \in R$ is in $(b R)_{a}$ if and only if $c / b \in R^{\prime}$.

Proof. This was proved in [8, Lemma 1] under the assumption that $R$ is Noetherian. However, this assumption was not used in the proof, so the same proof shows the present lemma.

Definition 2.4. Let $B=\left(b_{1}, \cdots, b_{k}\right) R$ be an ideal in a ring $R$, let $t$ be an indeterminate, and let $u=1 / t$. Then the Rees ring $\mathscr{R}=\mathscr{R}(R, B)$ of $R$ with respect to $B$ is defined to be the ring $\mathscr{R}=R\left[t b_{1}, \cdots, t b_{k}, u\right]$.

The elements in $\mathscr{R}$ are finite sums $\sum_{-p}{ }^{q} c_{2} t^{i}$, where $c_{i} \in B^{i}$ (with the convention that $B^{i}=R$, if $i \leqq 0$ ) and $p$ and $q$ are nonnegative integers, so $\mathscr{R}$ is a graded subring of $R[t, u]$. Also, it is clear that $u$ is not a divisor of zero in $\mathscr{R}$ and $u^{i} \mathscr{R} \cap R=B^{i}$, for all $i \geqq 0$. Finally, $\mathscr{R}$ is a Noetherian ring, if $R$ is.

Although the proof of the following lemma is straightforward, it is included to provide the reader some familiarity in working with Rees rings.

Lemma 2.5. (cf. [4, Theorem 3.7(4)].) Let $R, B$, and $\mathscr{R}$ be as in 2.4. Then $\left(u^{i} \mathscr{R}\right)_{a} \cap R=\left(B^{i}\right)_{a}$, for all $i>0$.

Proof. Let $x \in\left(u^{i} \mathscr{R}\right)_{a} \cap R$, so by (2.3), $x \in u^{i} \mathscr{R}^{\prime} \cap R$ and $t^{i} x \in \mathscr{R}^{\prime}$. Therefore, there exists $f_{1}, \cdots, f_{n} \in \mathscr{R}$ such that $\left(t^{i} x\right)^{n}+f_{1}\left(t^{i} x\right)^{n-1}+\cdots+$ $f_{n}=0$. Since $\mathscr{R}$ is a graded subring of $R[t, u]$ and each term on the left side of this equation is in $R[t, u]$, the nith component of the equation shows that $x \in\left(B^{i}\right)_{a}$ (by the fact that each $f_{h}$ is of the form $\sum_{-p}{ }^{q} r_{h j} t^{j}$, where $\left.r_{h j} \in B^{j}\right)$. For the opposite inclusion, if $x \in\left(B^{i}\right)_{a}$, then there exist $n \geqq 1$ and $r_{h} \in B^{i n}$ such that $x^{n}+r_{1} x^{n-1}+\cdots+$ $r_{n}=0, \quad$ so $\left(t^{i} x\right)^{n}+\left(t^{i} r_{1}\right)\left(t^{i} x\right)^{n-1}+\cdots+t^{i n} r_{n}=0$ and the $t^{i n} r_{h} \in \mathscr{R}$. Hence $t^{i} x \in \mathscr{R}^{\prime}$, so $x \in\left(u^{i} \mathscr{R}^{\prime} \cap \mathscr{R}\right) \cap R=\left(u^{i} \mathscr{R}\right)_{a} \cap R$.

Before stating the first theorem, further terminology and a brief summary of some known facts concerning these definitions will be given. 
Definition 2.6. An ideal $B$ in a ring is said to be of the principal class in case $B$ can be generated by $h$ elements, where $h=$ height $B$.

The above definition is usually given only for ideals in a Noetherian ring, and in such a ring it is well-known that an ideal which can be generated by $h$ elements has height at most equal to $h$.

DeFINITION 2.7. An ideal $B$ in a ring is height unmixed in case every prime divisor $P$ of $B$ is such that height $P=$ height $B$.

For example, an $R$-sequence in a locally Macaulay ring is height unmixed $[4,25.6]$.

Definition 2.8. An integral domain $R$ satisfies the altitude formula in case the following condition holds: For each finitely generated integral domain $A$ over $R$, and for each prime ideal $P$ in $A$, altitude $A_{P}+\operatorname{trd}(A / P) /(R /(P \cap R))=$ altitude $R_{P \cap R}+\operatorname{trd} A / R$, where $\operatorname{trd} D / C$ denotes the transcendence degree of the quotient field of an integral domain $D$ over the quotient field of its subdomain $C$.

Definition 2.9. A local ring $R$ is quasi-unmixed in case, for each minimal prime ideal $z$ in the completion of $R$, depth $z=$ altitude $R$. A Noetherian ring $R$ is locally quasi-unmixed in case, for each prime ideal $p$ in $R, R_{p}$ is quasi-unmixed.

The following remark lists the facts concerning these last two definitions which are needed in this paper.

REMARK 2.10. The following statements hold for a Noetherian ring $R$ :

2.10.1. If $R$ is an integral domain, then $R$ satisfies the altitude formula if and only if $R$ is locally quasi-unmixed [10, Theorem 3.6].

2.10.2. If $R$ is locally quasi-unmixed and $p \subset q$ are prime ideals in $R$, then height $q=$ height $p+$ height $q / p$ and $R / p$ is locally quasiunmixed (by $[4,34.5]$ ).

2.10.3. If $R \subseteq A \leqq C$ are integral domains such that $R$ satisfies the altitude formula, $A$ is finitely generated over $R$, and $C$ is integral over $A$, then both $A$ and $C$ satisfy the altitude formula and, for each prime ideal $p$ in $C$, height $p=$ height $p \cap A$ [10, Corollary 3.7 and Theorem 3.8].

A number of well-known relationships between a Noetherian 
integral domain and its integral closure continue to hold between a Noetherian ring and its integral closure. Since these facts are not so well-known, those needed in the remainder of this paper will be given in a remark prior to their first use. In particular, the following two facts are used in the proof of 2.12 below.

REMARK 2.11. Let $R^{\prime}$ be the integral closure of a Noetherian ring $R$ in its total quotient ring $T$.

2.11.1. If $I$ is an ideal in $T$, then $R /(I \cap R) \subseteq R^{\prime} /\left(I \cap R^{\prime}\right) \subseteq$ $(R /(I \cap R))^{\prime} 2.2$ [13, Remark 2.2].

2.11.2. Let $b$ be a nonunit nonzero-divisor in $R^{\prime}$. Then $b R^{\prime}$ is a finite intersection of height one primary ideals, and if $p^{\prime}$ is a prime divisor of $b R^{\prime}$, then height $p^{\prime}=1$ and there exists only one minimal prime ideal $z^{\prime}$ in $R^{\prime}$ such that $z^{\prime} \subseteq p^{\prime}$ [13, Proposition 2.13, Corollary 2.12, Definition (p. 213), and Proposition 2.7(1)].

The following theorem holds, in particular, for regular local rings, for locally Macaulay rings, and for complete local domains, since such rings are locally quasi-unmixed.

THEOREM 2.12. Let $R$ be a locally quasi-unmixed Noetherian ring, and let $B$ be an ideal of the principal class in $R$ 2.6. Then, for each integer $i>0,\left(B^{i}\right)_{a}$ is height unmixed 2.7 .

Proof. Since (0) is the only height zero ideal of the principal class in $R$, and since $\left(0^{i}\right)_{a}=\operatorname{Rad} R$ is height unmixed, it may be assumed that height $B>0$.

Let $\mathscr{R}=\mathscr{R}(R, B) 2.4$, fix $i>0$, let $p^{\prime}$ be a prime divisor of $u^{i} \mathscr{R}^{\prime} 2.2$, and let $p=p^{\prime} \cap \mathscr{R}$ and $P=p \cap R$. Then height $p^{\prime}=1$ and there exists only one minimal prime ideal $z^{\prime}$ in $\mathscr{R}^{\prime}$ such that $z^{\prime} \subset p^{\prime}$ 2.11.2. Let $z^{*}=z^{\prime} \cap \mathscr{R}$ and $z=z^{*} \cap R$, so $z+B \subseteq P$ (since $\left.u \in p^{\prime}\right)$. Let $\mathscr{T}$ be the total quotient ring of $\mathscr{R}$. Then, since $\mathscr{T}$ is the total quotient ring of $R[u], z^{\prime}=z \mathscr{T} \cap \mathscr{R}^{\prime}$ and $z$ is a minimal prime ideal in $R$. Therefore, $R / z \subseteq \mathscr{R} / z^{*} \subseteq \mathscr{R}^{\prime} / z^{\prime} \subseteq\left(\mathscr{R} / z^{*}\right)^{\prime}$ 2.11.1. Also, $R / z$ is locally quasi-unmixed 2.10 .2 , so $R / z$ satisfies the altitude formula 2.10.1. Therefore, with $q=p / z^{*}=\left(p^{\prime} / z^{\prime}\right) \cap\left(\mathscr{R} / z^{*}\right)$, height $q=2.10 .3$ height $p^{\prime} / z^{\prime}=1$, and height $q+\operatorname{trd}\left(\left(\mathscr{R} / z^{*}\right) / q\right) /((R / z) /(q \cap$ $(R / z)))=$ height $q \cap(R / z)+\operatorname{trd}\left(\mathscr{R} / z^{*}\right) /(R / z)$. Hence, since $\mathscr{R} / z^{*} \cong$ $\mathscr{R}(R / z,(B+z) / z)$ [18, Lemma 1.1], and since $q \cap(R / z)=P / z$, trd $(\mathscr{R} / p) /(R / P)=$ height $P / z=2.10 .2$ height $P \geqq$ height $B$ (since $B \subseteq$ $P)$. On the other hand, $\operatorname{trd}(\mathscr{R} / p) /(R / P) \leqq$ height $B$ (since $\mathscr{R}$ is generated by height $B+1$ elements over $R$ and $u \in p)$. Therefore height $P=$ height $B$. Hence, since $p^{\prime}$ is an arbitrary prime divisor 
of $u^{i} \mathscr{R}^{\prime}$ and $P=p^{\prime} \cap R$, since $\left(B^{i}\right)_{a}=2.5\left(u^{i} \mathscr{R}\right)_{a} \cap R=2.3 u^{i} \mathscr{R}^{\prime} \cap$ $\mathscr{R} \cap R$, and since height $B=$ height $B^{i}=$ height $\left(B^{i}\right)_{a},\left(B^{i}\right)_{a}$ is height unmixed.

The following remark lists two facts for future reference.

REMARK 2.13. Let $R, B$, and $\mathscr{R}$ be as in 2.12 and its proof.

2.13.1. The prime divisors of $\left(B^{i}\right)_{a}$ are the ideals $p^{\prime} \cap R$, where $p^{\prime}$ is a prime divisor of $u \mathscr{R}^{\prime}$.

2.13.2. Let $B=\left(b_{1}, \cdots, b_{k}\right) R$ (height $B=k$ ), and let $p$ be a prime divisor of $\left(u^{i} \mathscr{R}\right)_{a}$, where $i>0$. Then height $p=1, t b_{j} \notin p(j=1, \cdots$, $k$ ), and the residue classes modulo $p$ of the $t b_{j}$ are algebraically independent over $R /(p \cap R)$.

Proof. 2.13.1 was shown in the proof of 2.12.

2.13.2. Let $z_{0}^{*}$ be a minimal prime ideal in $\mathscr{R}$ such that $z_{0}^{*} \subset p$ and height $p=$ height $p / z_{0}^{*}$, let $z_{0}=z_{0}^{*} \cap R$, and let $P=p \cap R$. Then, as in the proof of 2.12 , the following statements hold: $z_{0}$ is a minimal prime ideal in $R$; $R / z_{0}$ satisfies the altitude formula; $\operatorname{trd}\left(\mathscr{R} / z_{0}^{*}\right) /\left(R / z_{0}\right)=1$; and, height $P / z_{0}=$ height $P=$ height $B$ (since $p=p^{\prime} \cap \mathscr{R}$ for some prime divisor $p^{\prime}$ of $u \mathscr{R}^{\prime} 2.3$ ). Therefore, applying the altitude formula for $p / z_{0}^{*}$ over $R / z_{0}$, it follows that height $p=$ height $p / z_{0}=1$ (since it was shown in the proof of 2.12 that $\operatorname{trd}(\mathscr{R} / p) /(R / P)=$ height $B$ ).

Corollary 2.14. Let $R$ and $B$ be as in 2.12, let $n>0$, and let $I$ be an ideal in $R$ such that $\left(b_{1}^{n}, b_{2}^{n}, \cdots, b_{k}^{n}\right) R \cong I \subseteq\left(B^{n}\right)_{a}$, where $B=$ $\left(b_{1}, \cdots, b_{k}\right) R$ (height $B=k$ ). Then, for all integers $i>0,\left(I^{i}\right)_{a}$ is height unmixed.

Proof. It is readily seen that, for each $i \geqq 1,\left(B^{n i}\right)_{a} \subseteq\left(\left(b_{1}^{n i}, b_{2}^{n i}\right.\right.$, $\left.\left.\cdots, b_{k}^{n i}\right) R\right)_{a}$, so $\left(B^{n i}\right)_{a} \subseteq\left(I^{i}\right)_{a} \subseteq\left(\left(\left(B^{n}\right)_{a}\right)^{i}\right)_{a}=\left(B^{n i}\right)_{a}$ (since $I \rightarrow I_{a}$ is a semiprime operation on the set of ideals in $R$ [6]). Therefore, the conclusion follows from 2.12 .

CoROLlaRY 2.15. Let $R$ be as in 2.12, let $p$ be a prime ideal in $R$, and let $A$ be a finitely generated integral domain over $R / p$. Then, for each ideal $B$ of the principal class in $A$ and for each integer $i>0,\left(B^{i}\right)_{a}$ is height unmixed.

Proof. This follows from 2.12 and the fact that $A$ is locally 
quasi-unmixed (by 2.10.2, 2.10.1, and 2.10.3).

DEFINITION 2.16. A ring $R$ satisfies the first chain condition for prime ideals (f.c.c.) in case every maximal chain of prime ideals in $R$ has length equal to the altitude of $R . \quad R$ is catenary is case, for each pair of prime ideals $p \subset q$ in $R,(R / p)_{q / p}$ satisfies the f.c.c.

REMARKs 2.17.1. It follows from [12, Corollary 3.13] and [11, Corollary 2.8] (resp., [12, Theorem 3.9]) that if a Noetherian ring $R$ satisfies the f.c.c. (resp., a Noetherian domain $R$ is catenary), then, for each nonmaximal prime ideal $p$ in $R, R_{p}$ is quasi-unmixed.

2.17.2. It follows from 2.12 that if $R$ is a complete local ring which satisfies the f.c.c. (so $R$ is quasi-unmixed $[4,34.4]$ ), then, for each ideal $B$ of the principal class in $R, R / B_{a}$ is an unmixed local ring (that is, every prime divisor $p$ of zero in (the completion of) $R / B_{a}$ is such that depth $p=$ altitude $R / B_{a}$ ). It is an open problem if the same result holds for noncomplete quasi-unmixed local rings.

COROLlaRy 2.18. Let $R$ be either a Noetherian ring which satisfies the f.c.c., or a Noetherian domain which is catenary, and let $B$ be an ideal of the principal class in $R$. Then, either $\left(B^{i}\right)_{a}$ is height unmixed, for all $i>0$, or the imbedded prime divisors of $\left(B^{i}\right)_{a}$ are maximal ideals in $R$.

Proof. This follows immediately from 2.12, 2.17.1, and the fact that $\left(B^{i}\right)_{a} R_{S}=\left(B^{i} R_{S}\right)_{a}$, where $S$ is a multiplicatively closed set in $R(0 \notin S)$.

Corollary 2.19. Let $R$ and $B$ be as in 2.12, and let $I$ be an ideal in $R$ such that $I$ is not contained in any minimal prime divisor of $B$. Then, for all $i>0$ and $n>0, B^{i}: I^{n} \subseteq\left(B^{i}\right)_{a}$.

Proof. Since $\left(B^{i}\right)_{a}$ is height unmixed 2.12, since $\operatorname{Rad}\left(B^{i}\right)_{a}=\operatorname{Rad} B$, and since $I$ is not contained in any minimal prime divisor of $B$, it follows that $B^{i}: I^{n} \subseteq\left(B^{i}\right)_{a}: I^{n}=\left(B^{i}\right)_{a}$.

The last corollary to 2.12 shows, in particular, that the conclusion of 2.12 holds in the integral closure of $R$.

CoROllary 2.20. Let $R$ be as in 2.12, and let $S$ be a ring such that $R \leqq S \leqq R^{\prime}$ 2.2. Then, for all ideals $B$ of the principal class in $S$ and for all integers $i>0,\left(B^{i}\right)_{a}$ is a finite intersection of primary ideals and is height unmixed. 
Proof. As in the proof of 2.12 , it may be assumed that $B \neq(0)$. Let $B=\left(b_{1}, \cdots, b_{k}\right) S$ be an ideal of the principal class in $S$ (height $B=k)$, and let $A=R\left[b_{1}, \cdots, b_{k}\right]$. Then $A$ is Noetherian and $B^{\prime}=$ $\left(b_{1}, \cdots, b_{k}\right) A$ is an ideal of the principal class in $A$ (since $S$ is integral over $A$ ). Further, $A$ is locally quasi-unmixed (since, if $P$ is a prime ideal in $A$ and $p=P \cap R$, then, with $Q=R_{p}$ and $L=A_{(R-p)}$ and with ${ }^{*}$ denoting completion, $Q^{*} \cong L^{*} \cong Q^{* \prime},\left(A_{P}\right)^{*} \cong L^{*}{ }_{P L^{*}}$, and, if $z$ is a minimal prime ideal in $L^{*}$ such that $z \subseteq P L^{*}$, then $Q^{*} /\left(z \cap Q^{*}\right)=$ (say) $C \subseteq L^{*} / z \subseteq C^{\prime} 2.11 .1$, so height $P L^{*} \geqq$ height $P L^{*} / z=$ (since $C$ is a complete local domain) height $p Q^{*} /\left(z \cap Q^{*}\right)=$ (since $R$ is locally quasi-unmixed) height $p Q^{*} \geqq$ (by integral dependence) height $P L^{*}$ ). Therefore, it may be assumed to begin with that $A=R$ and $B=B^{\prime} S$, where $B^{\prime}$ is an ideal of the principal class in $R$.

Let $\mathscr{R}=\mathscr{R}\left(R, B^{\prime}\right)$ and $\mathscr{S}=\mathscr{R}(S, B)$ 2.4, so $\mathscr{R} \subseteq \mathscr{S} \subseteq \mathscr{R}^{\prime}$. Fix $i>0$. Then, by the proof of 2.12, if $p^{\prime}$ is a prime divisor of $u^{i} \mathscr{R}^{\prime}$, then height $p^{\prime} \cap \mathscr{R}=1$, so height $p^{\prime} \cap \mathscr{S}=1$. Therefore by 2.3, $\left(u^{i} \mathscr{S}\right)_{a}=u^{i} \mathscr{R}^{\prime} \cap \mathscr{S}$ is height unmixed and is a finite intersection of primary ideals (since $u^{i} \mathscr{R}^{\prime}$ is 2.11.2). Hence $\left(B^{i}\right)_{a}=$ $\left(u^{i} \mathscr{S}\right)_{a} \cap S 2.5$ is a finite intersection of primary ideals. Also, for each minimal prime ideal $z$ in $S, S / z$ is integral over $R /(z \cap R)$ and $R /(z \cap R)$ satisfies the altitude formula, by 2.10 .2 and 2.10 .1 , so $S / z$ satisfies the altitude formula 2.10.3. Therefore, as in the last paragraph of the proof of 2.12 , it follows that $\left(B^{i}\right)_{a}$ is height unmixed.

A strong converse of 2.12 is given in 2.29 below. To prove 2.29, a number of preliminary results are needed, at least two of which are of some interest in themselves (2.22 and 2.24).

The following remark, which is needed for 2.22 and 2.24 below, gives some additional information on the properties of the integral closure of a Noetherian ring.

REMARK 2.21. Let $R, R^{\prime}$, and $b$ be as in 2.11.2, and let $S$ be a ring such that $R \cong S \subseteq R^{\prime}$. Assume $b \in S$ and $b S=(b S)_{a}$. Then the following statements hold:

2.21.1. $b S$ is a finite intersection of height one primary ideals, and if $p$ is a prime divisor of $b S$, then every $p S_{p}$-primary ideal $q$ is principal and $q=p^{i} S_{p}$, for some $i>0$, and $S_{p} /\left(\operatorname{Rad} S_{p}\right)$ is a discrete Archimedian valuation ring; in particular, by 2.3 this holds for $b R^{\prime}$ [13, Corollary 2.11 and Propositions 2.7(1) and 2.7(2)].

2.21.2. There is a one-to-one correspondence between the prime divisors $p$ of $b S$ and the prime divisors $p^{\prime}$ of $b R^{\prime}$ such that $p$ and $p^{\prime}$ correspond if and only if $S_{p}=R_{p^{\prime}}^{\prime}$. (This follows from $S_{p} \subseteq$ 
$R_{(S-p)}^{\prime} \leqq\left(S_{p}\right)^{\prime}=S_{p}$ (by [13, Remark 2.2, Proposition 2.7(3), and Corollary 2.11]), and from the fact tinat $p^{\prime} \cap S$ is a prime divisor of $b S$ [13, Theorem 2.15].)

Lemma 2.22. Let $b$ be a nonzero-divisor in the integral closure $R^{\prime}$ of a Noetherian ring $R$, and let $z$ be a minimal prime ideal in $R^{\prime}$. Assume $(z, b) R^{\prime} \neq R^{\prime}$. Then there exists a height one prime ideal $p$ in $R^{\prime}$ such that $(z, b) R^{\prime} \cong p$.

Proof. Let $T$ be the total quotient ring of $R$ and let $N=\operatorname{Rad}$ $T$, so $N \subseteq R^{\prime}$. Then $A=R /(N \cap R) \subseteq C=R^{\prime} / N \subseteq A^{\prime} 2.11 .1$, so $C^{\prime}=$ $A^{\prime}$ is a direct sum of $g$ Krull domains, where $g$ is the number of minimal prime ideals in $R$. Also, $\left(b R^{\prime}\right)_{a}=b R^{\prime} 2.3$, hence (clearly) $N \subseteq b R^{\prime}$, and, by [13, Lemma 2.4(3)] $b R^{\prime} / N=\left(b R^{\prime} / N\right)_{a}$. Therefore, by 2.21.1, $I=b R^{\prime} / N$ is a finite intersection of primary ideals and, for each prime divisor $p$ of the principal ideal $I, C_{p}$ is a discrete Archimedian valuation ring.

Let $z^{\prime}$ be the minimal prime ideal in $C^{\prime}$ such that $z^{\prime} \cap C=z / N$. Then, since $C^{\prime}$ is a direct sum of Krull domains and $\left(I, z^{\prime}\right) C^{\prime} \neq C^{\prime}$ (since $\left.(b, z) R^{\prime} \neq R^{\prime}\right)$, there exists a prime ideal $p^{\prime}$ in $C^{\prime}$ such that $p^{\prime}$ is a prime divisor of the principal ideal $I C^{\prime}$ and $z^{\prime} \subseteq p^{\prime}$; and then height $p^{\prime}=1$ 2.11.2. Therefore, by the one-to-one correspondence given in 2.21.2, height $p^{\prime} \cap C=1$ and $z / N$ is the only minimal prime ideal in $C$ which is contained in $p^{\prime} \cap C$ (since $C_{p^{\prime}}^{\prime}=C_{p^{\prime} \cap C}$ ). Let $p$ be the prime ideal in $R^{\prime}$ such that $p / N=p^{\prime} \cap C$. Then $z$ is the only minimal prime ideal in $R^{\prime}$ which is contained in $p$, so height $p=1$, and $(z, b) R^{\prime} \subseteq p$.

In 2.28(a) below an example is given to show that the above result fails to hold for a nonintegrally closed Noetherian ring, even if the ring is a complete local ring.

Corollary 2.23. Let $B$ be an ideal in a Noetherian ring $R$, and let $\mathscr{R}=\mathscr{R}(R, B)$. Then, for each minimal prime ideal $z$ in $R$ such that $B+z \neq R$, there exists a height one prime ideal $p^{\prime}$ in $\mathscr{R}^{\prime}$ such that $\left(z^{\prime}, u\right) \mathscr{R}^{\prime} \leqq p^{\prime}$, where $z^{\prime}=z \mathscr{T} \cap \mathscr{R}^{\prime}$ with $\mathscr{T}$ the total quotient ring of $\mathscr{R}$.

Proof. Note first that $\mathscr{T}$ is the total quotient ring of $R[u]$, so $z \mathscr{T}$ and $z^{\prime}$ are minimal prime ideals. Therefore, by 2.22 it suffices to prove that $\left(z^{\prime}, u\right) \mathscr{R}^{\prime} \neq \mathscr{R}^{\prime}$. For this, suppose that $\left(z^{\prime}, u\right) \mathscr{R}^{\prime}=\mathscr{R}^{\prime}$. Then $u+z^{\prime}$ is a unit in $\mathscr{R}^{\prime} / z^{\prime}$, so, by integral dependence, $u+\left(z^{\prime} \cap \mathscr{R}\right)$ is a unit in $\mathscr{R} /\left(z^{\prime} \cap \mathscr{R}\right)$. However, $\mathscr{R} /\left(z^{\prime} \cap \mathscr{R}\right) \cong \mathscr{R}(R / z,(B+z) / z)$ [18, Lemma 1.1], is $(B+z) / z=R / z$, hence $B+z=R$; contradiction. 
Therefore $\left(z^{\prime}, u\right) \mathscr{R}^{\prime} \neq \mathscr{R}^{\prime}$.

2.24 below is closely related to D. Rees' Valuation Theorem, and its proof uses a number of ideas and methods developed by him in [14], [15], [16], and [17].

Proposition 2.24. Let $R, B, \mathscr{R}$, and $\mathscr{R}^{\prime}$ be as in 2.23, and let $p_{1}^{\prime}, \cdots, p_{d}^{\prime}$ be the prime divisors of $u \mathscr{R}^{\prime}$. Fix $j(1 \leqq j \leqq d)$. Then $p_{j}^{\prime} \cap R$ is contained in a prime divisor of $\left(B^{i}\right)_{a}$, for all large $i$.

Proof. Let $C=\mathscr{R}^{\prime} \cap \mathscr{R}[1 / u]\left(=\mathscr{R}^{\prime} \cap R[t, u]\right)$, so $\mathscr{R}$ is a graded subring of $C$ and each homogeneous element in $C$ may be written in the form $b t^{n+i}=b t^{n} / u^{i}$, where $b \in B^{n}$ and $b t^{n} \in u^{i} \mathscr{R}^{\prime} \cap \mathscr{R}=\left(u^{i} \mathscr{R}\right)_{a}$. Also, $\left(u^{i} C\right)_{a}=u^{i} C$, for all $i>0$, since if $b t^{n}$ is a homogeneous element in $\left(u^{i} C\right)_{a}$, then $b t^{n+i} \in C^{\prime} \cap C[t]=C$ (since $C^{\prime}=\mathscr{R}^{\prime}$ and $\mathscr{R} \leqq C \leqq$ $\mathscr{R}[t]$ ), so $b t^{n} \in u^{i} C$, hence $\left(u^{i} C\right)_{a} \subseteq u^{i} C$ (since each is a homogeneous ideal); and the opposite inclusion is clear. Therefore, there exists a one-to-one correspondence between the prime divisors of $u^{i} C$ and $u^{i} \mathscr{R}^{\prime}$ 2.21.2 (so the prime divisors of $u^{i} C$ are the ideals $p_{j}=p_{j}^{\prime} \cap C$ $(j=1, \cdots, d)$, since the prime divisors of $u^{i} \mathscr{R}^{\prime}$ are the same as the prime divisors of $u \mathscr{R}^{\prime}$, for all $i>0$ ). Moreover, with $V_{j}=C_{p_{j}}$, the ideals $p_{j} V_{j}$ are principal 2.21.1, so define $e_{j}$ by $u V_{j}=p_{i}{ }^{e_{j}} V_{j}(j=1$, $\cdots, d)$. Now, $u^{i} C \cap R=u^{i} \mathscr{R}^{\prime} \cap C \cap \mathscr{R} \cap R=\left(B^{i}\right)_{a} 2.3$ and 2.5, for all $i>0$. Hence an element $r \in R$ is in $\left(B^{i}\right)_{a}=u^{i} C \cap R$ if and only if $r \in u^{i} V_{j} \cap C \cap R(j=1, \cdots, d)$. Therefore, for $j=1, \cdots, d$ and $r \in R$, define $v_{j}(r)=e_{j} i$ in case $r \in u^{i} V_{j} \cap C, \notin u^{i+1} V_{j} \cap C$; so $r \in\left(B^{i}\right)_{a}$ if and only if $v_{j}(r) \geqq e_{j} i(j=1, \cdots, d)$ (see 2.21.1).

Now fix $j$, say $j=1$, and let $x$ be a homogeneous element in $\bigcap\left\{u V_{j} \cap C ; j=2, \cdots, d\right\}$ such that $x \notin p_{1}$; say $x=r t^{n}$ (for some $r \in R$ ), so, for all $s \geqq 1, v_{j}\left(r^{s}\right)=v_{j}\left(u^{n s} x^{s}\right) \geqq s(n+1) e_{j}(j=2, \cdots, d)$ and $v_{1}\left(r^{s}\right)=v_{1}\left(u^{n s} x^{s}\right)=s n e_{1}$. Therefore $r^{s} \in\left(B^{s n}\right)_{a}, \notin\left(B^{s n+1}\right)_{a}$. Now, for $y \in P=p_{1} \cap R=p_{1}^{\prime} \cap R, v_{1}\left(y^{s e_{1}}\right) \geqq s e_{1}$, so $v_{j}\left(y^{s e_{1}} r^{s}\right) \geqq s(n+1) e_{j}(j=1$, $\cdots, d)$, hence $y^{s e_{1}} r^{s} \in\left(B^{s(n+1)}\right)_{a}$. Therefore, for $h=1, \cdots, s$ (and for $s \geqq 1),\left(B^{s n+h}\right)_{a}: y^{s e_{1}} R \neq\left(B^{s n+h}\right)_{a}$, so $\left(B^{s n+h}\right)_{a}: y R \neq\left(B^{s n+h}\right)_{a}$. It follows that $P$ is contained in a prime divisor of $\left(B^{i}\right)_{a}$, for all $i \geqq n^{2}+n+1$ (take $s \geqq n+1)$.

Corollary 2.25. Let $R, B, \mathscr{R}, \mathscr{R}^{\prime}$, and $p_{1}^{\prime}, \cdots, p_{d}^{\prime}$ be as in 2.24 . Then the following statements hold:

2.25.1. If $\left(B^{n}\right)_{a}$ has an imbedded prime divisor, for some $n>0$, then $\left(B^{i}\right)_{a}$ has an imbedded prime divisor, for all large $i$.

2.25.2. The ideals which are maximal (resp., minimal) in $\left\{p_{j}^{\prime} \cap R\right.$; 
$j=1, \cdots, d\}$ are maximal (resp., minimal) prime divisors of $\left(B^{i}\right)_{a}$, for all large $i$.

2.25.3. If $p_{j}^{\prime} \cap R$ is a maximal ideal in $R$, for some $j=1, \cdots, d$, then $p_{j}^{\prime} \cap R$ is a prime divisor of $\left(B^{i}\right)_{a}$, for all large $i$.

Proof. Since $u^{i} \mathscr{R}^{\prime} \cap R=\left(B^{i}\right)_{a}$, by 2.3 and 2.5 , if $\left(B^{n}\right)_{a}$ has an imbedded prime divisor $p$, for some $n>0$, then $p_{j}^{\prime} \cap R=p$, for some $j=1, \cdots, d$, so 2.25.1 follows from 2.24. 2.25.2 follows from 2.24 and the fact that $u^{i} \mathscr{R}^{\prime} \cap R=\left(B^{i}\right)_{a}$, since each $p_{j}^{\prime} \cap R$ contains a minimal prime divisor of $\left(B^{i}\right)_{a}$, for all $i>0$. Finally, 2.25.3 follows immediately from 2.25.2.

With $R^{*}$ the completion of a local domain $R$, the following statements are equivalent [10, Proposition 3.5]: There exists a height one maximal ideal in $R^{\prime}$; there exists a height one maximal ideal in $R^{* \prime}$; there exists a minimal prime ideal $z^{*}$ in $R^{*}$ such that depth $z^{*}=1$. Using this, 2.22, and [13, Remark 2.2 and Theorem 2.15], it can be proved (using a number of facts concerning the relationships between a local ring $R$ and its completion together with the fact that if $z$ is a minimal prime ideal in $R$, then

$$
R / z=(R /(\operatorname{Rad} R)) /(z /(\operatorname{Rad} R))
$$

that the result continues to hold for a local ring $R$. This fact is used in the proof of the following result.

Lemma 2.26. Let $\left(R^{*}, M^{*}\right)$ be the completion of a local ring $(R, M)$, and assume that there exists a minimal prime ideal $z$ in $R^{*}$ such that depth $z=1$. Then, for each ideal $B$ in $R$ such that height $B>0, M$ is a prime divisor of $\left(B^{i}\right)_{a}$, for all large $i$.

Proof. Let $B$ be an ideal in $R$ such that height $B>0$, let $\mathscr{R}^{*}=\mathscr{R}\left(R^{*}, B R^{*}\right)$, and let $p^{*}$ be a height one prime ideal in $\mathscr{R}^{* \prime}$ such that $\left(z \mathscr{T}^{*} \cap \mathscr{R}^{* \prime}, u\right) \mathscr{R}^{* \prime} \cong p^{*} 2.23$, where $\mathscr{T}^{*}$ is the total quotient ring of $\mathscr{R}^{*}$. Then $z \subset(z, B) R^{*} \leqq p^{*} \cap R^{*}$, so $p^{*} \cap R^{*}=M^{*}$. Let $q^{*}=p^{*} \cap \mathscr{R}^{*}$, let $\mathscr{R}=\mathscr{R}(R, B)$, and let $q=q^{*} \cap \mathscr{R}$. Then $M=q \cap R$, so $\mathscr{R}_{q}$ is a dense subspace of $\mathscr{R}_{q^{*}}^{*}[11$, Lemma 3.2]. Now there exists a height one maximal ideal in $\left(\mathscr{R}_{q^{*}}\right)^{\prime}$ (since $\mathscr{R}_{q^{*}} \subseteq$ $\left(\mathscr{R}^{* \prime}\right)_{\left(\mathscr{C}^{*}-q^{*}\right)} \subseteq\left(\mathscr{R}_{q^{*}}^{*}\right)^{\prime}[13$, Remark 2.2]), so there exists a height one maximal ideal in the integral closure of the completion of $\mathscr{R}_{q^{*}}^{*}$ (by the comment preceding this lemma). Therefore, since $\mathscr{R}_{q}$ is a dense subspace of $\mathscr{R}_{q^{*}}^{*}$, there exists a height one maximal ideal, say $N$, in $\mathscr{R}_{q}^{\prime}$ (again by the comment preceding this lemma). Hence, since 
$u \in N$, since $\mathscr{R}_{q} \subseteq \mathscr{R}_{(\mathscr{R}-q)}^{\prime} \subseteq \mathscr{R}_{q}^{\prime}$, and since $\left(u \mathscr{R}_{(\mathscr{R}-q)}^{\prime}\right)_{a}=u \mathscr{R}_{(\mathscr{R}-q)}^{\prime}[13$, Lemma 2.4(4)], it follows from 2.21.2 that there exists a height one prime ideal $p$ in $\mathscr{R}^{\prime}$ such that $p \cap \mathscr{R}=q$. Therefore, $p \cap R=M$, so, for all large $i, M$ is a prime divisor of $\left(B^{i}\right)_{a}$ 2.25.3.

It is known [3, Theorem 1] that if $p$ is a prime ideal in a Noetherian ring $R$, then there exist at most a finite number of prime ideals $q$ in $R$ such that $p \subset q$, height $q / p=1$, and height $q>$ height $p+1$. This fact is needed in the proof of the following proposition.

Proposition 2.27. Let $z$ be a minimal prime ideal in the completion $\left(R^{*}, M^{*}\right)$ of a local ring $(R, M)$, and let depth $z=d<m=$ altitude $R$. Then, for each $j=0,1, \cdots, m-d$, there exist infinitely many ideals $B_{j}$ of the principal class in $R$ such that height $B_{j}=$ $d+j$ and $M$ is a prime divisor of $\left(B_{j}^{i}\right)_{a}$, for all large $i$.

Proof. By 2.26 it may be assumed that $d>1$. Let $\mathscr{P}_{1}$ be the set of prime ideals $p$ in $R^{*}$ such that $z \subset p$ and height $p / z=1<$ height $p$. Then $\mathscr{P}_{1}$ is a finite set [3, Theorem 1], and $M \notin \mathscr{P}_{1}$ (since $d>1)$. Therefore, let $b_{1} \in M$ such that $b_{1} \notin \cup\left\{p \cap R ; p \in \mathscr{P}_{1}\right\} \cup U_{0}$, where $U_{0}$ is the union of the minimal prime ideals in $R$. Then $b_{1} R$ is an ideal of the principal class in $R$, and, by the principal ideal theorem in $R^{*} / z$, every minimal prime divisor of $\left(b_{1}, z\right) R^{*}$ has height one.

Let $p_{1}, \cdots, p_{s}$ be the minimal prime divisors of $\left(z, b_{1}\right) R^{*}$, so height $p_{i}=1$ and depth $p_{i}=d-1$ (since $R^{*} / z$ satisfies the f.c.c. $[4,34.4])$. If $d>2$, then let $\mathscr{P}_{2}$ be the set of prime ideals $p^{\prime}$ in $R$ such that, for some $i=1, \cdots, s, p_{i} \subset p^{\prime}$ and height $p^{\prime} / p_{i}=1<$ height $p^{\prime}-1$. Then $\mathscr{P}_{2}$ is a finite set [3, Theorem 1] and $M \notin \mathscr{P}_{2}$ (since $d>2$ ). Therefore, there exists $b_{2} \in M$ such that $b_{2} \notin \cup\left\{p^{\prime} \cap\right.$ $\left.R ; p^{\prime} \in \mathscr{P}_{2}\right\} \cup U_{1}$, where $U_{1}$ is the union of the minimal prime divisors of $b_{1} R$. It follows that $\left(b_{1}, b_{2}\right) R$ is an ideal of the principal class in $R$ and every minimal prime divisor of $\left(z, b_{1}, b_{2}\right) R^{*}$ has height equal to two (by the principal ideal theorem in $\left.R^{*} /\left(z, b_{1}\right) R^{*}\right)$. Therefore, after a finite number of repetitions, it is seen that there exists an ideal $\left(b_{1}, \cdots, b_{d-1}\right) R$ of height $d-1$ in $R$ such that every minimal prime divisor of $I^{*}=\left(z, b_{1}, \cdots b_{d-1}\right) R^{*}$ has height equal to $d-1$ (and depth equal to one).

Now let $b_{d}, \cdots, b_{d+j}(0 \leqq j \leqq m-d)$ in $M$ such that $B=\left(b_{1}, \cdots\right.$, $\left.b_{d-1}, b_{d}, \cdots, b_{d+j}\right) R$ is an ideal of the principal class in $R$ (of height $d+j)$. Let $\mathscr{R}=\mathscr{R}(R, B)$ and $\mathscr{R}^{*}=\mathscr{R}\left(R^{*}, B R^{*}\right)$, and let $p^{\prime}$ be a height one prime divisor of $\left(u, z^{\prime}\right) \mathscr{R}^{* \prime} 2.23$, where $z^{\prime}=z \mathscr{T}^{*} \cap \mathscr{R}^{* \prime}$ and $\mathscr{T}^{*}$ is the total quotient ring of $\mathscr{R}^{*}$. Then $(z, B) R^{*} \leqq p^{\prime} \cap \mathscr{R}^{*}$ and $(z, B) R^{*}$ is $M^{*}$-primary (since depth $I^{*}=1$ and $b_{d}$ is not in any 
minimal prime divisor of $I^{*}$ ). Therefore $p^{\prime} \cap R^{*}=M^{*}$. Hence, as in the proof of 2.26 , there exists a height one prime ideal $p$ in $\mathscr{R}^{\prime}$ such that $u \in p$ and $p \cap R=M$. Therefore, $M$ is a prime divisor of $\left(B^{i}\right)_{a}$, for all large $i 2.25$. Finally, there are infinitely many such $B$ (for each $j=0,1, \cdots, m-d$ ) by the infinite number of choices of the elements $b_{d}, \cdots, b_{d+j}$.

In view of 2.26 , it may be thought that 2.27 can be sharpened to: If a local ring $R$ is not quasi-unmixed, then every ideal $B$ of the principal class in $R$ such that height $B=$ altitude $R-1$ satisfies $\left(B^{i}\right)_{a}: M \neq\left(B^{i}\right)_{a}$, for all large $i$. However, this is not true, as is shown by (b) in the following example.

EXAMPLE 2.28. (a) It will first be shown that there exists a complete local ring $L^{*}$ such that there is a nonzero-divisor $c$ in $L^{*}$ and a minimal prime ideal $q^{*}$ in $L^{*}$ such that $1<\operatorname{depth} q^{*}=($ say) $d<$ altitude $L^{*}$ and $\left(c, q^{*}\right) L^{*}$ is a nonmaximal prime ideal such that height $\left(c, q^{*}\right) L^{*}>1$. Namely, let $(R, M)$ be as in [4, Example 2 , pp. 203-205] in the case $m>0$, so the integral closure $R^{\prime}$ of $R$ is a regular domain with exactly two maximal ideals, $N$ and $N^{\prime}$, such that height $N=m+1$ and height $N^{\prime}=r+m+1$, and $N \cap N^{\prime}=M$. Also, $N=\left(x, y_{1}, \cdots, y_{m}\right) R^{\prime}$ and $N^{\prime}=\left(x-1, y_{1}, \cdots, y_{m}, z_{1}, \cdots, z_{r}\right) R^{\prime}$ $\left(x, y_{i}\right.$, and $z_{j}$ as in [4]). Let $P=P^{\prime} \cap R$, where $P^{\prime}=\left(x, y_{1}, \cdots, y_{m-1}\right) R^{\prime}$, so height $P=m$, depth $P=1$, and $R^{\prime} / P^{\prime}=R / P$ is a discrete Archimedian valuation ring (so $\left(P, y_{m}\right) R=M$ ). Let $P^{* \prime}$ be a height one prime ideal in $R^{\prime}$ such that $P^{* \prime} \nsubseteq N$, and let $P^{*}=P^{* \prime} \cap R$, so $P^{*}$ is a height one prime ideal in $R$ such that depth $P^{*}=m+r$ and $P^{*} \nsubseteq P$. Let $K=P \cap P^{*}$, let $T_{1}, \cdots, T_{d-1}(d>1)$ be indeterminates, let $Q=R\left[T_{1}, \cdots, T_{d-1}\right]_{\left(M, T_{1}, \cdots, T_{d-1}\right)}$, and let $L=Q / K Q$. Then $L$ is a local ring with two minimal prime ideals, $q=P Q / K Q$ and $q^{\sharp}=P^{*} Q / K Q$, and depth $q=d$ while depth $q^{*}=m+r+d-1$. Let $"=M Q / K Q$, so " is a prime ideal in $L$ such that height $n=m+r$, depth $n=$ $d-1$, and ${ }_{n}=(q, c) L$, where $c=y_{m}+K Q$. Let $L^{*}$ be the completion of $L$. Then $q^{*}=q L^{*}$ is a minimal prime ideal in $L^{*}$ such that depth $q^{*}=d$ (since $R / P$ is a discrete Archimedian valuation ring implies that $Q / P Q$ is a regular local ring such that altitude $Q / P Q=d$, so $L^{*} / q^{*} \cong Q^{*} / P Q^{*} \cong\left(Q / P Q^{*}\right)$ is a regular local ring (where $*$ denotes completion)). Also, ${ }^{*}={ }^{*} L^{*}$ is a prime ideal such that height ${ }^{*}=$ $m+r$ and depth $.^{*}=d-1$ (since $R / M$ is a field implies that $Q / M Q$ is a regular local ring of altitude equal to $d-1$, so $L^{*} / 2^{*} \cong Q^{*} / M Q^{*} \cong$ $(Q / M Q)^{*}$ is a regular local ring). Finally, $\varkappa^{*}=\left(c, q^{*}\right) L^{*}$.

(b) There exists a local ring $(L, \ldots)$ and an ideal $B$ of the principal class in $L$ such that $L$ is not quasi-unmixed, height $B=$ altitude 
$L-1$, and $\left(B^{i}\right)_{a}: m=\left(B^{i}\right)_{a}$, for all $i>0$. Namely, let the notation be as in (a), and, for notational convenience, let $m=r=d-1=1$ (so altitude $L=3$ ). Let $y=y_{1}, z=z_{1}$, and $T=T_{1}$, and let ${ }^{\circ}$ denote residue classes modulo $K Q$. Also, in (a) choose $P^{* \prime}=(x-1) R^{\prime}$. Then $B=\left(y^{\circ}, z^{\circ}\right) L$ is of the principal class in $L$ (and height $B=2$, although height $\left.B L^{\prime}=1\right)$. Let $\mathscr{R}=\mathscr{R}(R, B)$, and let $p^{\prime}$ be a height one prime divisor of $\left(u, q^{\prime}\right) \mathscr{R}^{\prime} 2.22$, where $q^{\prime}=q \mathscr{T} \cap \mathscr{R}^{\prime}$ and $\mathscr{T}$ is the total quotient ring of $\mathscr{R}$. Then $R / q \subseteq \mathscr{R} /\left(q^{\prime} \cap \mathscr{R}\right)=$ (say) $\mathscr{S} \subseteq$ $\mathscr{R}^{\prime} / q^{\prime} \cong \mathscr{T} / q \mathscr{T}$, and $\mathscr{R}^{\prime} / q^{\prime}=\mathscr{S}^{\prime}$ (since $\operatorname{Rad} L=(0)$ implies that $\mathscr{R}^{\prime}$ is a direct sum of two Krull domains). Also, $\mathscr{S} \cong \mathscr{R}(L / q,(B+q) / q)$ [18, Lemma 1.1], and $L / q \cong(R / P)[T]_{(M / P, T)}$ is a regular local ring of altitude equal to two such that $(B+q) / q \cong\left(y^{\circ}, q\right) L / q$ (since $M=$ $(P, y) R$ implies that $z^{\circ}+q$ is in the ideal generated by $y^{\circ}+q$ in $L / q)$. Let $p^{*}=\left(p^{\prime} / q^{\prime}\right) \cap \mathscr{S}$, and let $p_{1}=p^{*} \cap(L / q)$. Then, since $L / q$ satisfies the altitude formula, height $p^{*}+t^{*}=$ height $p_{1}+1$, where $t^{*}=\operatorname{trd}\left(\mathscr{S} / p^{*}\right) /\left((L / q) / p_{1}\right) \leqq 1$ (since $(B+q) / q$ is a principal ideal); and height $p^{*}=12$ 2.10.3. Therefore, height $p_{1} \leqq 1$ (so height $p_{1}=1$ ), and so $p^{\prime} \cap L \neq \ldots$ (the maximal ideal in $L$ ). Therefore, it follows that no prime divisor of $u \mathscr{R}^{\prime}$ which contains $q^{\prime}$ contracts in $L$ to m. Similarly, no prime divisor of $u \mathscr{R}^{\prime}$ which contains $q^{\sharp} \mathscr{T} \cap \mathscr{R}^{\prime}$ contracts to $m$ (because altitude $L / q^{\sharp}=3$ and $L / q^{\sharp}$ satisfies the altitude formula (since $P^{* \prime} \nsubseteq N$ in (a))). Therefore, since $u^{i} \mathscr{R}^{\prime} \cap L=\left(B^{i}\right)_{a}$, it follows that $\left(B^{i}\right)_{a}: \mu=\left(B^{i}\right)_{a}$, for all $i>0$. Finally, $L$ is not quasiunmixed, by (a).

We can now prove the converse of 2.12. In fact, the following result holds.

THEOREM 2.29. The following statements are equivalent for a Noetherian ring $R$ :

2.29.1. $R$ is locally quasi-unmixed.

2.29.2. For all ideals $B$ of the principal class in $R,\left(B^{i}\right)_{a}$ is height unmixed, for all $i>0$.

2.29.3. For all ideals $B$ of the principal class in $R$ such that height $M / B=1$, for some maximal ideal $M$ in $R,\left(B^{i}\right)_{a}: M=\left(B^{i}\right)_{a}$, for infinitely many $i>0$.

Proof. 2.29.1 implies 2.29.2, by 2.12 , and it is clear that 2.29 .2 implies 2.29.3. Finally, if $R$ is not locally quasi-unmixed, then there exists a maximal ideal $M$ in $R$ such that $R_{M}=$ (say) $L$ is not quasiunmixed [7, Lemma 2.5]. Therefore, in the completion of $L$, there exists a minimal prime ideal $z$ such that depth $z=d<m=$ height $M$. If $d>1$, then let $\mathscr{P}_{1}, \cdots, \mathscr{P}_{d-1}$ and $U_{0}, \cdots, U_{d-2}$ be as in the proof of 2.27, and for $j=1, \cdots, d-1$, instead of choosing $b_{j} \in M L$, $\notin \cup\left\{p \cap L ; p \in \mathscr{P}_{j}\right\} \cup U_{j-1}$, rather, choose $b_{j} \in M, \notin \cup\left\{p \cap R ; p \in \mathscr{P}_{j}\right\} \cup$ 
$W_{j-1}$, where $W_{j-1}$ is the union of the minimal prime divisors of $\left(b_{1}\right.$, $\left.\cdots, b_{j-1}\right) R$. Then choose $b_{d}, \cdots, b_{m-1}$ in $M$ such that $B=\left(b_{1}, \cdots, b_{m-1}\right) R$ is an ideal of the principal class in $R$ (and height $B=m-1$ ), so $B L$ is an ideal of the principal class in $L$, and $\left(B^{i} L\right)_{a}: M L \neq\left(B^{i} L\right)_{a}$, for all large $i$ (by 2.27 and its proof). Therefore, since $\left(B^{i}\right)_{a} L=\left(B^{i} L\right)_{a}$, $\left(B^{i}\right)_{a}: M \neq\left(B^{i}\right)_{a}$, for all large $i$. If $d=1$, then for each ideal $B$ of the principal class in $R$ such that $B \subset M$ and height $B=m-1$, $\left(B^{i}\right)_{a}: M \neq\left(B^{i}\right)_{a} 2.26$ (since $\left.\left(B^{i}\right)_{a} L=\left(B^{i} L\right)_{a}\right)$. Thus 2.29.3 implies 2.29.1.

REMARK 2.30. If $B=\left(b_{1}, \cdots, b_{k}\right) R$ is an ideal in a ring $R$, then, for each integer $n>0,\left(\left(b_{1}^{n}, b_{2}^{n}, \cdots, b_{k}^{n}\right) R\right)_{a}=\left(B^{n}\right)_{a}$ (see the proof of $2.14)$, so it follows from 2.29 that a Noetherian ring $R$ is locally quasi-unmixed if and only if, for each ideal $B$ of the principal class in $R, B_{a}$ is height unmixed.

COROLLARY 2.31. The following statements are equivalent for a local ring $(R, M)$ :

2.31.1. $R$ is quasi-unmixed.

2.31.2. For each ideal $B$ of the principal class in $R,\left(B^{i}\right)_{a}$ is height unmixed, for all $i>0$.

2.31.3. For each ideal $B$ of the principal class in $R, B_{a}$ is height unmixed.

2.3ิ1.4. For each ideal $B$ of the principal class in $R$ such that height $B=$ altitude $R-1,\left(B^{i}\right)_{a}: M=\left(B^{i}\right)_{a}$, for infinitely many $i$.

Proof. This is clear by 2.29 and 2.30 .

Of course, 2.28(b) shows that the equivalent statements in 2.31 are not equivalent to: There exists an ideal $B$ of the principal class in $R$ such that height $B=$ altitude $R-1$ and $\left(B^{i}\right)_{a}: M=\left(B^{i}\right)_{a}$, for all large $i$.

If follows from 2.30 that the condition: For each ideal $B$ of the principal class in $R, B_{a}$ is height unmixed; is inherited by finite integral extension rings of $R$, by factor domains of $R$, and by finitely generated extension domains of factor damains of $R$ (since such rings are locally quasi-unmixed).

3. Some applications. The results in this section are, essentially, corollaries to 2.12. However, a number of definitions and some remarks concerning the definitions are needed before stating the results. Since giving these definitions and remarks in $\S 2$ would have created a diversion from our main goal in that section, it was decided to present this material in a separate section of this paper. 
Definition 3.1. Let $B$ be an ideal in a local ring $(R, M)$. The analytic spread of $B$ is defined to be the nonnegative integer $l(B)=$ $\operatorname{deg} F(i, B)+1$, where $F(i, B)=\operatorname{dim}_{R / M} B^{i} / M B^{i}$ (so $F(i, B)$ is a polynomial in $i$, for all large $i$ [5, Theorem 1, p. 148]).

DEFINITION 3.2. If $q$ is an $M$-primary ideal in a local ring $(R, M)$, then $e(q)$ denotes the multiplicity of $q[22$, p. 294].

Definition 3.3. Let $B \cong C$ be ideals in a ring $R$. Then $B$ is said to be a reduction of $C$ in case there exists a positive integer $n$ such that $C^{n+1}=B C^{n} . \quad B$ is a minimal reduction of $C$ in case $B$ is minimal in the set of reductions of $C$.

REMARKS 3.4.1. If $B$ is an ideal in a local ring $(M, R)$, then height $B \leqq l(B) \leqq \operatorname{dim}_{R / M} B / M B$ [5, Lemma 4, p. 151].

3.4.2. If $B \subseteq C \subseteq D$ are ideals in a ring $R$ such that $B$ is a reduction of $C$ and $C$ is a reduction of $D$, then $B$ is a reduction of $D$ [5, Lemma 1, p. 146].

3.4.3. If $C$ is an ideal in a local ring $(R, M)$, then minimal reductions of $C$ exist [5, Theorem 1, p. 147]. If, moreover, $R / M$ is an infinite field, then the number of elements in a minimal base of a minimal reduction of $C$ is $l(C)$ [5, Theorem 1, p. 150].

3.4.4. If $B \subseteq C$ are ideals in a Noetherian ring $R$, then $B$ is a reduction of $C$ if and only if $B_{a}=C_{a}$ [4, Exercise 4, p. 34]. Therefore, if $B$ is a reduction of $C$, then $\operatorname{Rad} B=\operatorname{Red} C$, and it is easily shown [5, Theorem 1, p. 146] that $e\left(B R_{p}\right)=e\left(C R_{p}\right)$, for each minimal prime divisor $p$ of $B$ and of $C$. On the other hand, it is a deep result due to $\mathrm{D}$. Rees that if $B \cong C$ are $M$-primary ideals in a quasiunmixed local ring $(R, M)$ such that $e(B)=e(C)$, then $B$ is a reduction of $C$ [19, Theorem 3.2].

This last result of Rees has been generalized by E. Böger in [1, Theorem 1] and in [2, Theorem 1] (see 3.5 below). In [1] the proof of the generalized result is long and difficult, and in [2] it is still quite lengthy and deep. That a short and fairly easy proof can be given using 2.12 will now be shown. (It should be noted that, once the definitions are known, the proof of 2.12 is really not difficult.)

Proposition 3.5. Let $B \subseteq C$ be ideals in a quasi-unmixed local ring $(R, M)$, and assume that height $B=l(B) 3.1, \operatorname{Rad} B=\operatorname{Rad} C$, and $e\left(B R_{p}\right)=e\left(C R_{p}\right)$, for each minimal prime divisor $p$ of $B$. Then $B$ is a reduction of $C$ 3.3.

Proof. Assume first that $R / M$ is an infinite field, and let $X$ be 
a minimal reduction of $B$ 3.4.3. Then $X \subseteq B$ and $X_{a}=B_{a}$ 3.4.4. (so $\operatorname{Rad} X=\operatorname{Rad} B$ ), and the number of elements in a minimal base of $X$ is $l(B)$ 3.4.3. Therefore, $X$ is an ideal of the principal class and height $X=l(X)$ (since height $X=$ height $B=l(B)=\operatorname{dim}_{R / M} X / M X=$ (by 3.4.1) $l(X)$. Also, $e\left(X R_{p}\right)=e\left(B R_{p}\right)$, for each minimal prime divisor $p$ of $B$ 3.4.4. Therefore, it may be assumed to begin with that $B$ is an ideal of the principal class (since $X$ is). Then $B_{a}$ is height unmixed by hypothesis, [7, Lemma 2.5], and 2.12, and if $p$ is a minimal prime divisor of $B$, then $R_{p}$ is quasi-unmixed and $e\left(B R_{p}\right)=$ $e\left(C R_{p}\right)$, so $B R_{p}$ is a reduction of $C R_{p}$ [19, Theorem 3.2] (see 3.4.4), hence $\left(B R_{p}\right)_{a}=\left(C R_{p}\right)_{a}$ 3.4.4. Therefore, with $\mathscr{P}$ the set of minimal prime divisors of $B, C_{a} \supseteqq B_{a}=2.12 \bigcap\left\{B_{a} R_{p} \cap R ; p \in \mathscr{P}\right\}=\bigcap\left\{\left(B R_{p}\right)_{a} \cap\right.$ $R ; p \in \mathscr{P}\}=\bigcap\left\{\left(C R_{p}\right)_{a} \cap R ; p \in \mathscr{P}\right\} \supseteqq C_{a}$, so $B$ is a reduction of $C$ 3.4.4.

If $R / M$ is a finite field, then let $R^{*}=R[X]_{M R \mid X]}$, where $X$ is an indeterminate, and let $M^{*}=M R^{*}$. Then $\left(R^{*}, M^{*}\right)$ is a local ring such that $R^{*} / M^{*}$ is an infinite field, $B^{*}=B R^{*} \subseteq C R^{*}=C^{*}$, and height $B^{*}=$ height $B=l(B)=l\left(B^{*}\right)$, the last equality following from 3.1 and well-known facts concerning $F(i, B) . \quad$ Also $\operatorname{Rad} B^{*}=\operatorname{Rad} C^{*}$, and, for each minimal prime divisor $p^{*}$ of $B^{*}, p^{*}=p R^{*}$, for some minimal prime divisor $p$ of $B$, and then $e\left(B^{*} R_{p^{*}}^{*}\right)=e\left(B R_{p}\right)=e\left(C R_{p}\right)=$ $e\left(C^{*} R_{p^{*}}^{*}\right)$. Finaly, $R^{*}$ is quasi-unmixed [7, Lemma 2.7], so by the first paragraph of this proof, $B^{*}$ is a reduction of $C^{*}$. Therefore $\left(B^{*}\right)_{a}=$ $\left(C^{*}\right)_{a}$ 3.4.4. Now, since $R[X]$ is a graded ring and, for each ideal $I$ in $R, I R[X]$ is a homogeneous ideal, it is readily seen that $(I R)_{a} R[X]=$ $(I R[X])_{a}$, hence $(I R)_{a} R^{*}=\left(I R^{*}\right)_{a}$. Therefore

$$
B_{a}=B_{a} R^{*} \cap R=C_{a} R^{*} \cap R=C_{a},
$$

hence $B$ is a reduction of $C$ 3.4.4.

CoROLlary 3.6. Let $B$ be an ideal of the principai class in a locally quasi-unmixed Noetherian ring $R$, and let $C$ be an ideal in $R$ such that $B \leqq C, \operatorname{Rad} B=\operatorname{Rad} C$, and $e\left(B R_{p}\right)=e\left(C R_{p}\right)$, for all minimal prime divisors $p$ of $B$. Then $B$ is a reduction of $C$.

Proof. Let $p$ be a minimal prime divisor of $B$. Then, by hypothesis and [19, Theorem 3.2] $B R_{p}$ is a reduction of $C R_{p}$, so $\left(B R_{p}\right)_{a}=$ $\left(C R_{p}\right)_{a}$. Therefore, by 2.12 and as in the last sentence of the first paragraph of the proof of $3.5, B_{a}=C_{a}$, hence $B$ is a reduction of $C$.

Since $B$ is of the principal class in $R$ in 3.6, it can be shown (using 3.4.1 and 3.4.3) that $B$ is, in fact, a minimal reduction of $C$.

Corollary 3.7. Let $B \subseteq C$ be ideals in a locally quasi-unmixed 
Noetherian ring $R$, and assume that $\operatorname{Rad} B=\operatorname{Rad} C$ and $e\left(B R_{p}\right)=$ $e\left(C R_{p}\right)$, for all minimal prime divisors $p$ of $B$. If there exists an $n>0$ such that $B^{n}$ is integral over an ideal $I$ of the principal class in $R$ (that is, $I \leqq B^{n} \leqq I_{a}$ ), then $B$ is a reduction of $C$.

Proof. Note first that $\operatorname{Rad} I=\operatorname{Rad} B^{n}, I \subseteq B^{n}$, and, for each minimal prime divisor $p$ of $I, I R_{p} \subseteq B^{n} R_{p} \subseteq\left(I R_{p}\right)_{a}$, hence $e\left(I R_{p}\right)=$ $e\left(B^{n} R_{p}\right)$ 3.4.4. Therefore, by 3.6, $I$ is a reduction of $B^{n}$.

It will now be shown that $I$ is also a reduction of $C^{n}$. For this, let $p$ be a minimal prime divisor of $I$, and assume temporarily that $R_{p} / p R_{p}$ is an infinite field (this holds if $p$ is not a maximal ideal in $R)$. Then there exists an ideal $X$ such that $X$ is generated by a system of parameters $x_{1}, \cdots, x_{h}$ in $R_{p}, X \subseteq C R_{p}$, and $e(X)=e\left(C R_{p}\right)$ [22, Theorem 22, p. 294]. Therefere, $X$ is a reduction of $C R_{p}[19$, Theorem 3.2], so $X^{n}$ is a reduction of $C^{n} R_{p}$ and $X^{[n]}=\left(x_{1}^{n}, x_{2}^{n}, \cdots, x_{h}^{n}\right) R_{p}$ is readily seen to be a reduction of $X^{n}$, hence $X^{[n]}$ is a reduction of $C^{n} R_{p}$ 3.4.2. Thus $e\left(C^{n} R_{p}\right)=e\left(X^{[n]}\right)=([14$, Lemma 6.1] or [4, 24.5]) $n^{h} e(X)$. Likewise, $e\left(B^{n} R_{p}\right)=n^{h} e(Y)$, where $Y=\left(y_{1}, \cdots, y_{h}\right) R_{p}$ is such that $e(Y)=e\left(B R_{p}\right)$. Therefore, by hypothesis, $e\left(C^{n} R_{p}\right)=e\left(B^{n} R_{p}\right)$, so $e\left(C^{n} R_{p}\right)=e\left(I R_{p}\right)$. Hence, since $I \leqq C^{n}$ and $\operatorname{Rad} I=\operatorname{Rad} C^{n}, I$ is a reduction of $C^{n} 3.6$. If $R_{p} / p R_{p}$ is not an infinite field, then, as in the second paragraph of the proof of 3.5 , it is seen also in this case that $I$ is a reduction of $C^{n}$. Therefore, there exists an integer $i>0$ such that $I C^{n i}=\left(C^{n}\right)^{i+1}$, hence $C^{n i+n}=I C^{n i} \cong B^{n} C^{n i} \cong C^{n i+n}$, so $B C^{n i+n-1}=C^{n i+n}$, and so $B$ is a reduction of $C$.

COROLLARY 3.8. Let $R$ be either a Noetherian ring which satisfies the f.c.c. or a Noetherian domain which is catenary, and let $B$ be an ideal of the principal class in $R$. Assume that $C$ is an ideal in $R$ such that $B \subseteq C, \operatorname{Rad} B=\operatorname{Rad} C$, and $e\left(B R_{p}\right)=e\left(C R_{p}\right)$, for all minimal prime divisors $p$ of $B$. If no maximal ideal in $R$ is a prime divisor of $B_{a}$, then $B$ is a reduction of $C$.

Proof. If no maximal ideal in $R$ is a prime divisor of $B_{a}$, then $B_{a}$ is height unmixed 2.18 , and $R_{p}$ is quasi-unmixed, for all minimal prime divisors $p$ of $B$ 2.17.1. Therefore, that $B$ is a reduction of $C$ follows as in the last two sentences in the first paragraph of the proof of 3.5 .

Corollary 3.9. Let $R$ be as in 3.8, and let $B \cong C$ be ideals in $R$ such that $\operatorname{Rad} B=\operatorname{Rad} C$ and $e\left(B R_{p}\right)=e\left(C R_{p}\right)$, for all minimal prime divisors $p$ of $B$. Assume that there exists an integer $n>0$ such that $B^{n}$ is integral over an ideal $I$ of the principal class in $R$ and that no maximal ideal in $R$ is a prime divisor of $I_{a}$. Then 
$B$ is a reduction of $C$.

Proof. As in the proof of 3.7, for each minimal prime divisor $p$ of $I, e\left(I R_{p}\right)=e\left(B^{n} R_{p}\right)=e\left(C^{n} R_{p}\right)$ and $\left(I R_{p}\right)_{a}=\left(B^{n} R_{p}\right)_{a}=\left(C^{n} R_{p}\right)_{a}$ (since $R_{p}$ is quasi-unmixed 2.17.1). Therefore, since $I_{a}$ is height unmixed 2.18, $I_{a}=\left(B^{n}\right)_{a}=\left(C^{n}\right)_{a}$, as in the last sentence of the first paragraph of the proof of 3.5 , so $B^{n}$ is a reduction of $C^{n}$, hence $B$ is a reduction of $C$ (since $B \subseteq C$ ).

It can be proved that if instead of assuming that no maximal ideal is a prime divisor of $I_{a}$ in 3.9, rather, it is assumed that no maximal ideal is a prime divisor of $\left(B^{n i}\right)_{a}$, for some $i>0$, then again $B$ is a reduction of $C$.

To derive another corollary of 2.12, the following definition is needed.

Definition 3.10. A semi-local ring $R$ is analytically unramified in case there are no nonzero nilpotent elements in the completion of $R$.

Proposition 3.11. Let $R$ be a semi-local ring which is analytically unramified and locally quasi-unmixed. Then, for each ideal $B$ of the principal class in $R$, and for all large $n, B_{n}^{i}$ is height unmixed and $B_{n}^{i}=\left(B_{n}^{i}\right)_{a}$, for all $i>0$, where $B_{n}=\left(B^{n}\right)_{a}$.

Proof. Let $B$ be an ideal of the principal class in $R$. If $B=(0)$, then $B=\operatorname{Rad} R$ (since $R$ is analytically unramified), so $\left(B^{i}\right)_{a}=(0)=B^{i}$, for all $i>0$. Therefore, it may be assumed that $B \neq(0)$. Let $M_{1}$, $\cdots, M_{d}$ be the maximal ideals in $R$. Fix $j(1 \leqq j \leqq d)$ and let $M=$ $M_{j}$. Then, since $R_{M}$ is analytically unramified, it follows from [20, Theorem 2] that, for all large $n, I_{n}=\left(B^{n} R_{M}\right)_{a}$ is such that $I_{n}^{i}=\left(I_{n}^{i}\right)_{a}$, for all $i>0$. It follows that, for all large $n, B_{n}=\left(B^{n}\right)_{a}=\bigcap\left\{\left(B^{n}\right)_{a} R_{M_{n}} \cap\right.$ $R ; j=1, \cdots, d\}=\bigcap\left\{\left\{B^{n} R_{M_{j}}\right)_{a} \cap R ; j=1, \cdots, d\right\}$ is such that $B_{n}^{i}=$ $\left(B_{n}^{i}\right)_{a}$, for all $i>0$; and $\left(B_{n}^{i}\right)_{a}=\left(B^{n i}\right)_{a}$ is height unmixed 2.12.

REMARKS 3.12.1. If $R$ is a Noetherian ring which is locally analytically unramified and locally quasi-unmixed, then the same proof shows that the above result holds for each ideal $B$ of the principal class in $R$ such that $B$ is contained in only finitely many maximal ideals in $R$.

3.12.2. Note that $\operatorname{Rad} B_{n}=\operatorname{Rad} B$ and $B_{n} \subseteq B$ (for large $n$ [20, Lemma 3]), so 3.11 says that each ideal $B$ of the principal class contains an ideal $I$ such that $\operatorname{Rad} I=\operatorname{Rad} B$ and $I^{i}$ is height unmixed, for all $i>0$. 
One final application of 2.12 will be given in 3.13 below. To motivate the result, we note that it is known [22, Lemmas 5 and 6, pp. 401-402] that if $b_{1}, \cdots, b_{k}$ is an $R$-sequence in a Macaulay local ring $R$, then the following statements hold for $B=\left(b_{1}, \cdots, b_{k}\right) R$ : (a) $B^{i}$ is height unmixed, for all $i>0$; and, (b) $B^{i}: b_{j} R=B^{i-1}$, for all $i>0$ and $j=1, \cdots, k$. It was seen in 2.12 that an analogue of (a) holds in a locally quasi-unmixed Noetherian ring $R$ for an ideal $B$ of the principal class in $R .3 .13$ shows that an analogue of (b) is also true in this case.

Proposition 3.13. Let $R$ be a locally quasi-unmixed Noetherian ring, and let $B=\left(b_{1}, \cdots, b_{k}\right) R$ be an ideal of the principal class in $R$ (height $B=k>0$ ). Then, for each $i>0$ and $j=1, \cdots, k,\left(B^{i}\right)_{a}: b_{j} R=$ $\left(B^{i-1}\right)_{a}$.

Proof. Fix $j(1 \leqq j \leqq k)$, let $i>0$, and let $r \in\left(B^{i}\right)_{a}: b_{j} R$. Then $r b_{j} \in\left(B^{i}\right)_{a}$, so with $\mathscr{R}=\mathscr{R}(R, B)$ and as in the proof of $2.5 t^{i} r b_{j} \in$ $\mathscr{R}^{\prime}$, and $t r b_{j} \in \mathscr{R}$. Therefore, $t r b_{j} \in u^{i-1} \mathscr{R}^{\prime} \cap \mathscr{R}=\left(u^{i-1} \mathscr{R}\right)_{a}$, hence $r \in\left(u^{i-1} \mathscr{R}\right)_{a}: t b_{j} \mathscr{R}$. Now $\left(u^{i-1} \mathscr{R}\right)_{a}$ is height unmixed 2.13.2, so it follows from 2.13.2 that $r \in\left(u^{i-1} \mathscr{R}\right)_{a} \cap R=\left(B^{i-1}\right)_{a}$. For the opposite inclusion, if $r \in\left(B^{i-1}\right)_{a}$, then $r \in\left(u^{i-1} \mathscr{R}\right)_{a}$, so $t r b_{j} \in\left(u^{i-1} \mathscr{R}\right)_{a}$, hence $t^{i} r b_{j} \in \mathscr{R}^{\prime} 2.3$, so $r b_{j} \in u^{i} \mathscr{R}^{\prime} \cap R=\left(B^{i}\right)_{a}$, and so $r \in\left(B^{i}\right)_{a}: b_{j} R$.

Definition 3.14. An element $b$ in an ideal $B$ in a ring $R$ is said to be a strongly superficial element for $B$ in case, for each integer $i>0, B^{i}: b R=B^{i-1}$.

A number of properties of strongly superficial elements are given in [21], but we only need the definition for this paper.

CoRollary 3.15. Let $R$ be a semi-local ring which is analytically unramified and locally quasi-unmixed, and let $B=\left(b_{1}, \cdots, b_{k}\right) R$ be an ideal of the principal class in $R$ (height $B=k>0$ ). Then, for each $j=1, \cdots, k$ and for all large $n, b_{j}^{n}$ is a strongly superficial element 3.14 for $\left(B^{n}\right)_{a}$.

Proof. Fix $j(1 \leqq j \leqq k)$, let $n$ be large (so with $B_{n}=\left(B^{n}\right)_{a}$, $B_{n}^{i}=\left(B_{n}^{i}\right)_{a} 3.11$, for all $\left.i>0\right)$. Therefore, for all $i>0, B_{n}^{i}: b_{j}^{n} R=3.11$ $\left(B^{n i}\right)_{a}: b_{j}^{n} R=3.14\left(B^{n i-n}\right)_{a}=3.11 B_{n}^{i-1}$.

\section{REFERENCES}

1. E. Böger, Eine Verallgemeinerung eines Multiplizitätensatzes von D. Rees, J. Algebra, 12 (1969), 207-215.

2. - Einige Bemerkungen zur Theorie der ganzalgebraischen Abhängigkeit von Idealen, Math. Ann., 185 (1970), 303-308. 
3. S. McAdam, Saturated chains in Noetherian rings. forthcoming.

4. M. Nagata, Local Rings, Interscience tracts 13, Interscience, New York, 1962.

5. D. G. Northcott and D. Rees, Reductions of ideals in local rings, Proc. Cambridge Philos. Soc., 50 (1954), 145-158.

6. J. W. Petro, Some results on the asymptotic completion of an ideal, Proc. Amer. Math. Soc., 15 (1964), 519-524.

7. L. J. Ratliff, Jr., On quasi-unmixed semi-local rings and the altitude formula, Amer. J. Math., 87 (1965), 278-284.

8. - Note on analytically unramified semi-local rings, Proc. Amer. Math. Soc., 17 (1966), 274-279.

9. - A characterization of analytically unramified semi-local rings and applications, Pacific J. Math., 27 (1968), 127-143.

10. - On quasi-unmixed local domains, the altitude formula, and the chain condition for prime ideals (I), Amer. J. Math., 91 (1969), 508-528.

11. On quasi-unmixed local domains, the altitude formula, and the chain condition for prime ideals (II), Amer. J. Math., 92 (1970), 99-144.

12. C Characterizations of catenary rings, Amer. J. Math., 93 (1971), 10701108.

13. - On prime divisors of the integral closure of a principal ideal, J. Reine Angew. Math., 255 (1972), 210-220.

14. D. Rees, Valuations associated with a local ring (I), Proc. London Math. Soc., 5 (1955), 107-128.

15. - Valuations associated with ideals (I), Proc. London Math. Soc., 6 (1956), $161-174$.

16. - Valuations associated with ideals (II), J. London Math. Soc., 31 (1956), 228-235.

17. - Valuations associated with ideals (II), J. London Math. Soc., 31 (1956), $221-228$.

18. - A note on form rings and ideals, Mathematika, 4 (1957), 51-60.

19. - 2-transforms of local rings and a theorem on multiplicities of ideals, Proc. Cambridge Philos. Soc., 57 (1961), 8-17.

20. M. Sakuma and H. Okuyama, On a criterion for analytically unramification of a local ring, J. Gakugei Tokushima Univ., 15 (1966), 36-38.

21. Merrilene Smith, Strongly superficial elements, forthcoming.

22. O. Zariski and P. Samuel, Commutative Algebra, Vol. II, Van Nostrand, New York, 1960.

Received September 19, 1973. Work on this paper was supported in part by the National Science Foundation, Grant NSF GP-28939-1.

University of CALifornia, Riverside 



\section{PACIFIC JOURNAL OF MATHEMATICS}

EDITORS

RICHARD ARENS (Managing Editor)

University of California

Los Angeles, California 90024

R. A. Beaumont

University of Washington

Seattle, Washington 98105

J. DugundJI

Department of Mathematics

University of Southern California

Los Angeles, California 90007

D. Gilbarg and J. Milgram

Stanford University

Stanford, California 94305

\section{ASSOCIATE EDITORS}

E. F. BECKENBACH

B. H. NeumanN

F. WOLF

K. YOSHIDA

\section{SUPPORTING INSTITUTIONS}

UNIVERSITY OF BRITISH COLUMBIA CALIFORNIA INSTITUTE OF TECHNOLOGY

UNIVERSITY OF CALIFORNIA

MONTANA STATE UNIVERSITY

UNIVERSITY OF NEVADA

NEW MEXICO STATE UNIVERSITY

OREGON STATE UNIVERSITY

UNIVERSITY OF OREGON

OSAKA UNIVERSITY

\author{
UNIVERSITY OF SOUTHERN CALIFORNIA \\ STANFORD UNIVERSITY \\ UNIVERSITY OF TOKYO \\ UNIVERSITY OF UTAH \\ WASHINGTON STATE UNIVERSITY \\ UNIVERSITY OF WASHINGTON \\ $\stackrel{*}{*} \stackrel{*}{*}{ }^{*}{ }^{*}{ }^{2}$ AMERICAN MATHEMATICAL SOCIETY \\ NAVAL WEAPONS CENTER
}




\section{Pacific Journal of Mathematics}

\section{Vol. 52, No. $1 \quad$ January, 1974}

David R. Adams, On the exceptional sets for spaces of potentials ............ 1

Philip Bacon, Axioms for the Čech cohomology of paracompacta ............ 7

Selwyn Ross Caradus, Perturbation theory for generalized Fredholm operators ..... 11

Kuang-Ho Chen, Phragmén-Lindelöf type theorems for a system of nonhomogeneous equations ............................ 17

Frederick Knowles Dashiell, Jr., Isomorphism problems for the Baire classes .......

M. G. Deshpande and V. K. Deshpande, Rings whose proper homomorphic images are right subdirectly irreducible . . . . . . . . . . . . . . . . . . . . . . . . .

Mary Rodriguez Embry, Self adjoint strictly cyclic operator algebras .............

Paul Erdős, On the distribution of numbers of the form $\sigma(n) / n$ and on some related

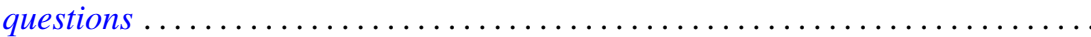

Richard Joseph Fleming and James E. Jamison, Hermitian and adjoint abelian

operators on certain Banach spaces ............................

Stanley P. Gudder and L. Haskins, The center of a poset .................. 85

Richard Howard Herman, Automorphism groups of operator algebras . . . ........

Worthen N. Hunsacker and Somashekhar Amrith Naimpally, Local compactness of families of continuous point-compact relations ....................

Donald Gordon James, On the normal subgroups of integral orthogonal groups ....

Eugene Carlyle Johnsen and Thomas Frederick Storer, Combinatorial structures in

loops. II. Commutative inverse property cyclic neofields of prime-power

order.

Ka-Sing Lau, Extreme operators on Choquet simplexes . . . . . . . . . . . . . . 129

Philip A. Leonard and Kenneth S. Williams, The septic character of 2, 3, 5 and $7 \ldots 143$

Dennis McGavran and Jingyal Pak, On the Nielsen number of a fiber map ........ 149

Stuart Edward Mills, Normed Köthe spaces as intermediate spaces of $L_{1}$ and

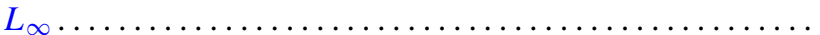

Philip Olin, Free products and elementary equivalence. .

Louis Jackson Ratliff, Jr., Locally quasi-unmixed Noetherian rings and ideals of the principal class.

Seiya Sasao, Homotopy types of spherical fibre spaces over spheres ...

Helga Schirmer, Fixed point sets of polyhedra ...

Kevin James Sharpe, Compatible topologies and continuous irreducible

representations.

Frank Siwiec, On defining a space by a weak base . . . . . . . . . . . . . . . 233

James McLean Sloss, Global reflection for a class of simple closed curves ....... 247

M. V. Subba Rao, On two congruences for primality . .

Raymond D. Terry, Oscillatory properties of a delay differential equation of even

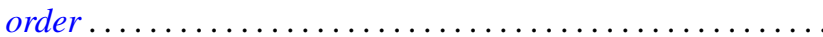

Joseph Dinneen Ward, Chebyshev centers in spaces of continuous functions . .

Robert Breckenridge Warfield, Jr., The uniqueness of elongations of Abelian

groups...

V. M. Warfield, Existence and adjoint theorems for linear stochastic differential

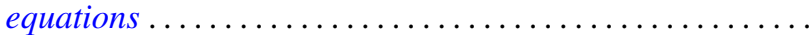

